\title{
Postoperative Apnea in a Neonate Following an Epidural Bolus Dose Through a High Thoracic Epidural Catheter
}

\author{
Jay Mathiasa, b, e, Deanna Couser ${ }^{\mathrm{c}}$, David P. Martin ${ }^{\mathrm{c}, \mathrm{d}}$, Joseph D. Tobias ${ }^{\mathrm{c}, \mathrm{d}}$
}

\begin{abstract}
Regional anesthesia is being used more frequently in the practice of pediatric anesthesia including neonates and infants. While generally safe and effective, adverse effects may occur related to catheter placement or its subsequent use. We present the rare occurrence of high motor blockade with apnea following the administration of a bolus dose of the local anesthetic agent, 2-chloroprocaine, into the thoracic epidural catheter of a 4-week-old, 2.2-kg former premature neonate. The patient had an epidural catheter that had been threaded from the caudal space to the thoracic level to provide analgesia following an abdominal surgical procedure. Subsequent investigation with a standard chest radiograph revealed a higher than intended placement of the epidural catheter (T4 instead of T8-10) which resulted in a transient high motor blockade with apnea. The epidural infusion was discontinued and assisted ventilation was provided by bag-valve-mask ventilation. Immediately, the heart rate and oxygen saturation returned to baseline values, and within $5 \mathrm{~min}$ the patient became more active, spontaneous ventilation resumed, and a strong cry was noted. The epidural catheter was removed and the remainder of the postoperative course was unremarkable. Adverse effects of epidural anesthesia in neonates are discussed and options for identifying the correct placement of a thoracic epidural catheter are reviewed.
\end{abstract}

Keywords: Epidural anesthesia; Apnea; Chloroprocaine

\section{Introduction}

Until the late 1980s, it had been proposed that neonates lacked

Manuscript submitted October 14, 2021, accepted November 8, 2021

Published online December 2, 2021

aHeritage College of Osteopathic Medicine, Dublin Campus, Dublin, OH, USA

bOhio University, Athens, OH, USA

'Department of Anesthesiology \& Pain Medicine, Nationwide Children's Hospital, Columbus, OH, USA

dDepartment of Anesthesiology \& Pain Medicine, The Ohio State University College of Medicine, Columbus, OH, USA

${ }^{\mathrm{e} C}$ Corresponding Author: Jay Mathias, Department of Anesthesiology \& Pain Medicine, Nationwide Children's Hospital, 700 Children's Drive, Columbus, OH 43205, USA. Email: jm870311@ohio.edu

doi: https://doi.org/10.14740/jmc3814 perception, localization, and memory of pain and that they experienced pain differently from the adult population [1]. For the majority of the 20th century, the physiological signs and behavioral responses that neonates showed in response to pain were at times dismissed thereby resulting in the undertreatment of pain associated with surgical procedures $[1,2]$. Neglected pain management in neonates resulted in short- and long-term physiological, behavioral, and cognitive complications that included impaired brain growth, attention-deficit disorder, poor executive function, poor socialization skills, and poor shortterm memory tasks $[1,2]$. This misunderstanding of neonatal pain was refuted by clinical trials that showed a reduction in stress hormone levels and metabolic responses to pain when neonates were given adequate anesthesia [1,2].

Effective pain management in neonates remains a priority in the neonatal intensive care unit (NICU) and the operating room (OR) settings as it has been shown to have the potential to improve various clinical outcomes in neonates $[1,2]$. Opioids, such as morphine and fentanyl, remain the mainstay treatment for severe pain; however, their use may be associated with adverse effects including respiratory depression and delayed gastrointestinal motility [2]. Epidural anesthesia has shown to be safe and effective in pediatrics, even in neonates $[3,4]$. Despite its promising efficacy in controlling postoperative pain, adverse effects may occur related to catheter placement or its subsequent use.

We present a 4-week-old, formerly preterm, 2.2-kg neonate who developed apnea after a bolus dose of the local anesthetic agent, 2-chloroprocaine, and was administered into a thoracic epidural catheter. Further investigation revealed the tip of the epidural catheter at a higher level than intended which resulted in a transient high motor blockade and apnea. Adverse effects of epidural anesthesia in neonates are discussed and options for identifying the correct placement of a thoracic epidural catheter are reviewed.

\section{Case Report}

\section{Investigations}

Review of this case and presentation in this format followed the guidelines of the Institutional Review Board of Nationwide Children's Hospital (Columbus, OH). The patient was a 4-week-old, 2.2-kg male neonate presented to the OR for ileostomy takedown. His past medical history was significant 
for preterm birth at 33 weeks gestation, meconium ileus resulting in small bowel obstruction requiring an ileostomy, and bronchopulmonary dysplasia requiring supplemental of $0.1 \mathrm{~L} /$ min oxygen. The patient was transported to the OR, and routine American Society of Anesthesiologists' monitors were placed. Baseline vital signs revealed a heart rate (HR) of 135 beats per minute (bpm), blood pressure of $86 / 40 \mathrm{~mm} \mathrm{Hg}$, a respiratory rate of 32 breaths/min, and an oxygen saturation of $99 \%$ on $0.1 \mathrm{~L} / \mathrm{min}$ of oxygen via nasal cannula. Following preoxygenation with $100 \%$ oxygen, anesthesia was induced with propofol $(3 \mathrm{mg})$ and fentanyl $(2 \mu \mathrm{g} / \mathrm{kg})$. Neuromuscular blockade was provided by rocuronium $(1 \mathrm{mg} / \mathrm{kg})$. A $3.0-\mathrm{mm}$ cuffed endotracheal tube was placed. Following anesthetic induction and endotracheal intubation, anesthesia was maintained on sevoflurane and the patient was positioned in the right lateral decubitus position.

The caudal epidural space was accessed through the sacral hiatus with an 18-gauge, 5-cm Epican ${ }^{\mathrm{TM}}$ Crawford needle. A 20-gauge styletted epidural catheter was threaded to the T10 level with ultrasound guidance. After negative aspiration for blood or cerebrospinal fluid, a test dose $(0.2 \mathrm{~mL}$ of $1.5 \%$ lidocaine with 1:200,000 epinephrine) was administered into the epidural catheter. No hemodynamic changes were observed, and an epidural infusion of $1.5 \% 2$-chloroprocaine and clonidine $(0.1 \mu \mathrm{g} / \mathrm{mL})$ was started at $2 \mathrm{~mL} / \mathrm{h}$ per standard clinical practice [4]. An additional $1 \mu \mathrm{g}$ of fentanyl was administered intraoperatively and anesthesia was maintained with sevoflurane (end-tidal concentration $0.2-1.2 \%$ ). There were no changes in the hemodynamic parameters during surgical stimulation. The surgical procedure lasted approximately 120 $\min$. There were no intraoperative concerns. At the conclusion of the surgical procedure, the patient's trachea was extubated in the OR and he was taken to the post anesthesia care unit (PACU).

While in the PACU, the epidural infusion rate was decreased to $1 \mathrm{~mL} / \mathrm{h}$ as the patient's blood pressure was slightly decreased. The patient was admitted to the NICU postoperatively. Postoperative analgesia was assessed using the face, legs, activity, cry, consolability (FLACC) scoring system and neonatal pain, agitation, and sedation scale (NPASS) scoring system. From postoperative day (POD) $0-1$, pain was managed with an epidural infusion of $1.5 \%$ 2-chloroprocaine with clonidine $(0.1 \mu \mathrm{g} / \mathrm{mL})$ at $1 \mathrm{~mL} / \mathrm{h}$ and intravenous acetaminophen $(10 \mathrm{mg} / \mathrm{kg})$ every $6 \mathrm{~h}$. Two supplemental doses of fentanyl ( 1 and $2 \mu \mathrm{g}$ ) were administered during the first 24 postoperative hours. On POD 2, the epidural dressing appeared to be leaking which required the catheter site to be cleaned and redressed. In addition, the patient was reported to be more irritable and required additional fentanyl ( $3 \mu \mathrm{g}$ total). A $1-\mathrm{mL}$ bolus of the $1.5 \%$ 2-chloroprocaine and clonidine $(0.1 \mu \mathrm{g} / \mathrm{mL})$ was administered through the epidural and the epidural infusion was increased to $2 \mathrm{~mL} / \mathrm{h}$. Minutes later, the patient developed apnea, a decrease in the HR, and a decrease in the oxygen saturation.

\section{Diagnosis}

After the administration of a bolus dose of 2-chloroprocaine

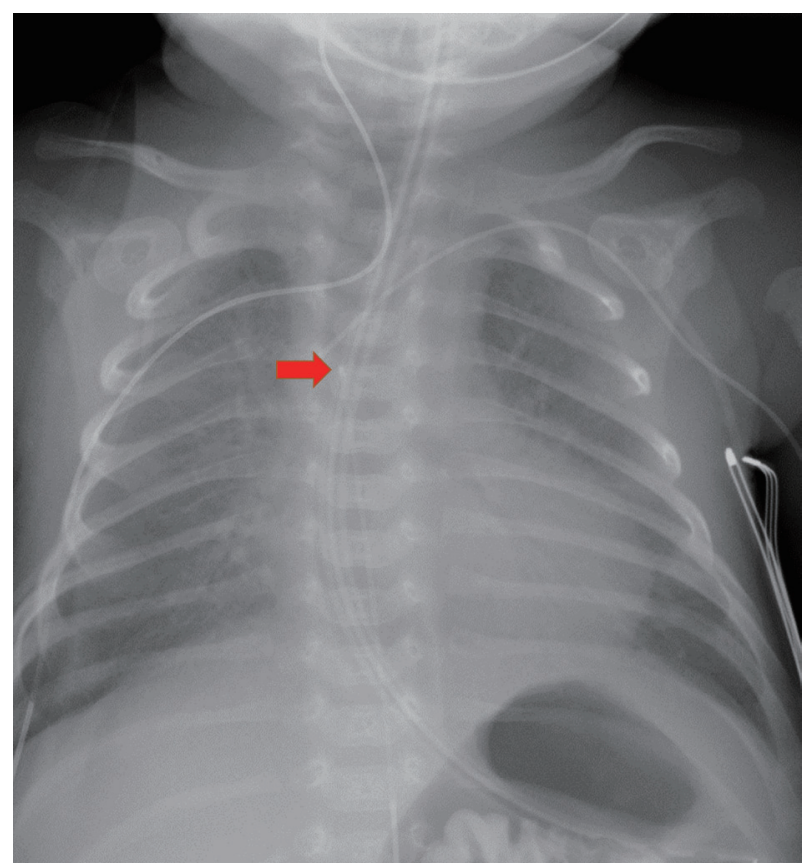

Figure 1. Chest radiograph demonstrating the tip of the epidural catheter at the T4 level (red arrow).

into the epidural catheter and the onset of apnea, a high motor block was suspected and confirmed by a radiograph (Fig. 1) which revealed the tip of the epidural catheter to be at T4.

\section{Treatment}

During this time, the patient's eyes were open, he was looking around, and was appearing to attempt to cry, but no sound was audible. Although there were respiratory efforts, no chest, diaphragmatic or extremity movements were noted. Positive pressure ventilation was administered by bag-valve-mask and the epidural infusion was discontinued. The HR and oxygen saturation immediately returned to baseline values. Within 5 min of stopping the epidural infusion, the patient became more active, spontaneous ventilation resumed, and a strong cry was noted. The patient was placed on supplemental oxygen, the epidural catheter was removed and postoperative analgesia provided by intravenous fentanyl delivered by nurse-controlled analgesia infusion.

\section{Follow-up and outcomes}

The remainder of the postoperative course was without adverse or unanticipated events. The patient's pain was routinely assessed with FLACC and NPASS scoring systems, and was adequately controlled with intravenous fentanyl. A change in clinical practice was instituted to routinely obtain and review a postoperative radiograph to identify the site of catheter placement when an epidural catheter is threaded from the caudal to thoracic space in neonates in the postoperative period. 


\section{Discussion}

Intravenous opioids have been the mainstay therapy for providing reliable postoperative analgesia in the pediatric population [2]. However, due to the immaturity of the central control of ventilation and reduced opioid clearance, infants and neonates are vulnerable to opioid-induced respiratory depression and its sequelae. Regional anesthesia, including epidural analgesia, has been shown to be a safe and effective option for intraoperative and postoperative analgesia in neonates and infants. Earlier tracheal extubation, reduced intraoperative opioid use, blunting of hormonal-metabolic stress response, earlier return of bowel function, decreased respiratory complications and shorter hospital stays are benefits to patients receiving epidural analgesia compared to systemic opioids $[5,6]$.

Despite its promising efficacy in controlling postoperative pain, adverse effects may occur related to catheter placement or an infusion of medications through an epidural catheter. Previous work has demonstrated the risk of local anesthetic systemic toxicity (LAST) when higher infusion rates are administered through lumbar catheters to achieve thoracic analgesia [7]. In order to provide effective analgesia and limit local anesthetic agent infusion rates, effective epidural anesthesia requires placement of the tip of the catheter near the dermatomes affected by the surgical procedure. This requires placement of the catheter at the thoracic dermatomes for thoracic and upper abdominal procedures.

Two common approaches to achieve this desired placement are threading the catheter from the caudal approach and direct placement into the thoracic epidural space $[8,9]$. The caudal approach was first described in 1933 by Meredith Campbell, using a single injection into the caudal space ("the caudal block") to provide postoperative analgesia in children undergoing urologic surgery [10]. Since its introduction, the safety and efficacy of caudal blockade has been well described and has expanded to include continuous epidural infusions. The feasibility of placing a thoracic epidural catheter from the caudal space was first introduced in 1988 by Bosenburg et al in a three-phase study including 14 human cadavers, 12 living piglets, and 20 neonates and infants, ranging in age from 4 weeks to 5 months and in weight ranged from 2.7 to $6.5 \mathrm{~kg}$ [9]. This technique was first investigated in human cadavers, followed by a demonstration of its efficacy in an animal model. Proof of concept was then demonstrated in a cohort of 20 neonates and infants, 19 of whom were able to have the catheter placed within one vertebral level of the desired position as confirmed by radiographic imaging. These results were reinforced by two subsequent investigators $[11,12]$. One potential limitation to these studies was that the target level for the epidural catheter was in the lower thoracic segments (T10-12) with limited data on placement at higher thoracic dermatomes.

When the decision is made to thread the catheter to the higher thoracic levels from the caudal space, ensuring appropriate placement is paramount to achieving successful analgesia and avoiding adverse effects. In the three previously mentioned studies, the distance to thread the catheter into the epidural space was determined by measuring externally from the skin puncture site in the caudal area to the desired verte- bral level. Despite measuring the distance of the catheter to be inserted, placement at the desired dermatome is not uniformly successful. It has been demonstrated that $32 \%$ or 28 of 86 caudally threaded thoracic epidural catheters were not at the desired level when subsequently evaluated using a standard radiograph [13]. Ten of the 28 inadequately placed epidural catheters were in the high thoracic or cervical region which could have led to an adverse effect similar to that noted in our patient, had radiographic confirmation not been obtained. During threading the catheter from the caudal approach, various issues may be encountered including catheter kinking, doubling back on itself, or meeting resistance. Although traditionally reserved for the adult population, these issues can be avoided by direct catheter placement at the thoracic level. Despite concerns expressed regarding the potential for complications with this practice, no difference in the incidence or severity of adverse effects has been noted when comparing direct thoracic epidural catheter placement with the technique of threading the catheter from the caudal space [14].

Various techniques are available to identify catheter location in the epidural space based on the type of catheter used and whether or not it is radio-opaque. Some catheters are reinforced with an internal wire coil, such as the type used in our patient, and are easily seen on a routine chest radiograph. Catheters that are not radio-opaque require the injection of contrast or a radio-opaque dye to identify their position on standard radiographs [15]. These techniques do not allow real time advancement of the catheter and would mandate catheter manipulation and repeating the radiograph if the appropriate location has not been achieved. Given that the preliminary clinical work has demonstrated that external measurement and blind placement is not always uniformly successful, other techniques are needed to ensure success. Fluoroscopy can be used in real time to confirm placement, rule out incorrect anatomic space, and predict analgesic coverage. However, such techniques are associated with ionizing radiation and the need for added equipment in the OR, additional cost, and additional time.

Other options suggested that may be effective in ensuring accurate placement of the tip of the epidural catheter include nerve stimulation and ultrasound-guided techniques. Electrical stimulation to document epidural catheter placement or guide its advancement was first described by Tsui et al in the adult population [16]. Catheter placement was considered to be in the correct position based on the presence or absence of truncal or limb movement to $1-10 \mathrm{~mA}$ of electrical stimulation. This work was later expanded to include the pediatric population and guide advancement of the catheter from the caudal to the thoracic epidural space [17].

Ultrasound has also been used as a modality to identify the location of the epidural catheter in various small studies and case reports [18-23]. In the largest study to date which included 25 patients ( 2 days to 5 months of age), the authors were able to detect the epidural catheter tip via ultrasound in 22 of the 25 patients [22]. Success in localization of the epidural catheter via ultrasound has been reported to be easier in patients less than 6 months of age because neuraxial structures are more superficial and the vertebrae are less ossified. Techniques that may increase the ability to identify the catheter in 
the epidural space include displacement of the dura mater by the injection of saline or the use of a hyperechoic stylet within the catheter $[23,24]$. Despite the growing popularity and reported accuracy of ultrasound guidance, limitations associated with this method include the need for a skilled provider, poor visualization of the catheter tip in the epidural space especially if the patient is older than 6 months of age, and inability to see local anesthetic spread. As a result, there have been reported cases of misplacement even with this technique [25].

Epidural catheters can be left in place for days after a given procedure in order to provide adequate analgesia. Despite the various methods used to secure the epidural catheter in place, Simpao and colleagues demonstrated that caudally threaded epidural catheters can migrate one or more vertebral levels after placement during the postoperative period [26]. In a study of 85 patients, catheter migration of $\geq 1$ vertebral level was noted in 54 patients $(64 \%)$ and 23 patients $(27 \%)$ had a catheter at the T4 level or higher. Catheter migration of two or more vertebral levels occurred more commonly in infants who weighed less than $6 \mathrm{~kg}$. Thus, continuous epidural catheter monitoring via postoperative imaging may be essential for safety and efficacy.

Although generally safe and effective, various adverse effects have been reported with epidural anesthesia in infants and children including those related to initial needle or catheter placement and those related to its subsequent use. Adverse effects related to placement including the potential for bleeding or direct neurologic damage are rare. There were no reports of hematoma formation or permanent neurologic injures in a 2018 review of neuraxial anesthesia from the Pediatric Regional Anesthesia Network (PRAN) [25]. More commonly, adverse effects are related to the medications used to provide epidural anesthesia including the local anesthetic agent or adjuncts such as opioids or $\alpha_{2}$-adrenergic agonists. Given the potential for LAST, strict attention to dosing guidelines is mandatory during epidural anesthesia, especially in neonates and infants [27].

We report an uncommon adverse effect of thoracic epidural anesthesia, apnea from high blockade following bolus dosing of epidural catheter with the local anesthetic agent, 2-chloroprocaine. Within minutes following the bolus dose, our patient developed apnea, a decrease in the heart rate, and a decrease in the oxygen saturation. During this time while respirations were absent (no diaphragmatic or thoracic movement), the patient's eyes were open, he was looking around, and attempting to cry, but no sound was audible. After assisted ventilation was provided by bag-valve-mask ventilation, a radiograph was obtained which revealed the tip of the epidural catheter to be at T4. Although high blockade is generally more common with spinal anesthesia, it can occur with epidural related to large doses or as was noted in our patient, high catheter placement (T4 versus the desired location of T8-10).

Our experience with this patient demonstrates another potential advantage of using the short-acting local anesthetic agent, 2-chloroprocaine, given its short half-life and duration of action, compared to other commonly used agents such as bupivacaine or ropivacaine. Especially in neonates, given the variable pharmacokinetics of the amide local anesthetic agents related to immaturity of the hepatic microsomal enzymes and decreased local anesthetic binding capacity, 2-chloroprocaine is being used more commonly to limit the incidence of LAST $[27,28]$. Although originally described to provide dense surgical anesthesia during herniorrhaphy using regional instead of general anesthesia, 2-chloroprocaine is now being used more commonly for continuous postoperative epidural infusions [3, $4,29,30]$. Its potential advantages over local anesthetic agents of the amide group are illustrated by a recent case report where LAST occurred following inadvertent systemic injection following injection of a thoracic epidural catheter (T7 level) in a 4-kg, 2-month-old infant [31]. Immediately after the injection of $4 \mathrm{~mL}$ of $3 \% 2$-chloroprocaine, the patient developed a wide complex bradycardia without evidence of atrial activity. While preparations were being made to initiate resuscitation, the event terminated spontaneously. The duration of the event was approximately $30 \mathrm{~s}$. A similar event was described following bolus dosing of a paravertebral catheter with 2-chloroprocaine in a 9-month-old infant [32]. This event and the one described in our patient demonstrate the potential advantage of a shortacting agent such as 2-chloroprocaine should adverse effects occur. In our patient, the high motor blockade and respiratory compromise (apnea) resolved in $5 \mathrm{~min}$ and required only support by bag-valve-mask ventilation instead of endotracheal intubation.

\section{Learning points}

Neuraxial anesthesia remains an important method of providing postoperative analgesia with specific advantages over systemic opioids and an excellent adverse effect profile even in neonates and infants. Although infrequent, adverse effects related to placement or subsequent use of the catheter may occur. We present the rare occurrence of high motor blockade following the administration of a bolus dose of the local anesthetic agent, 2-chloroprocaine, into a thoracic epidural catheter in a 4-week-old neonate. Subsequent radiologic investigation demonstrated that the tip of the epidural catheter was higher than anticipated. Although ultrasound is being used more commonly as the epidural catheter is being advanced from the caudal to the thoracic level, our anecdotal experience suggests that postoperative radiographic evaluation should also be considered. Our experience further reinforces the safety profile of 2-chloroprocaine and its potential advantages over the long acting local anesthetics of the amide class. Givens its rapid metabolism, the high motor blockade dissipated rapidly without the need for interventions beyond brief respiratory assistance with bag-valve-mask ventilation.

\section{Acknowledgments}

None to declare.

\section{Financial Disclosure}

This research did not receive any specific grant from funding agencies in the public, commercial, or not-for-profit sectors. 


\section{Conflict of Interest}

None to declare.

\section{Informed Consent}

Informed consent was obtained from a parent for anesthetic care and use of patient data for publication purposes. The patient information was deidentified for publication.

\section{Author Contributions}

JM performed the initial case review and manuscript preparation, literature review, and editing of subsequent revisions. DC and DPM provided clinical care and reviewed the manuscript. JDT contributed to literature review, manuscript writing and editing of the manuscript.

\section{Data Availability}

The data supporting the findings of this study are available from the corresponding author upon reasonable request.

\section{References}

1. Anand KJ, Hickey PR. Pain and its effects in the human neonate and fetus. N Engl J Med. 1987;317(21):13211329.

2. Hall RW, Anand KJ. Pain management in newborns. Clin Perinatol. 2014;41(4):895-924.

3. Veneziano G, Tobias JD. Chloroprocaine for epidural anesthesia in infants and children. Paediatr Anaesth. 2017;27(6):581-590.

4. Gibbs A, Kim SS, Heydinger G, Veneziano G, Tobias J. Postoperative analgesia in neonates and infants using epidural chloroprocaine and clonidine. J Pain Res. 2020;13:2749-2755.

5. Martin LD, Adams TL, Duling LC, Grigg EB, Bosenberg A, Onchiri F, Jimenez N. Comparison between epidural and opioid analgesia for infants undergoing major abdominal surgery. Paediatr Anaesth. 2019;29(8):835-842.

6. Berde C. Epidural analgesia in children. Can J Anaesth. 1994;41(7):555-560.

7. Berde CB. Convulsions associated with pediatric regional anesthesia. Anesth Analg. 1992;75(2):164-166.

8. Tobias JD, Lowe S, O'Dell N, Holcomb GW, 3rd. Thoracic epidural anaesthesia in infants and children. Can J Anaesth. 1993;40(9):879-882.

9. Bosenberg AT, Bland BA, Schulte-Steinberg O, Downing JW. Thoracic epidural anesthesia via caudal route in infants. Anesthesiology. 1988;69(2):265-269.

10. Campbell MF. Caudal anesthesia in children. J Urol. 1933;30:245-250.

11. Rasch DK, Webster DE, Pollard TG, Gurkowski MA.
Lumbar and thoracic epidural analgesia via the caudal approach for postoperative pain relief in infants and children. Can J Anaesth. 1990;37(3):359-362.

12. Gunter JB, Eng C. Thoracic epidural anesthesia via the caudal approach in children. Anesthesiology. 1992;76(6):935-938.

13. Valairucha S, Seefelder C, Houck CS. Thoracic epidural catheters placed by the caudal route in infants: the importance of radiographic confirmation. Paediatr Anaesth. 2002;12(5):424-428.

14. Polaner DM, Taenzer AH, Walker BJ, Bosenberg A, Krane EJ, Suresh S, Wolf C, et al. Pediatric Regional Anesthesia Network (PRAN): a multi-institutional study of the use and incidence of complications of pediatric regional anesthesia. Anesth Analg. 2012;115(6):1353-1364.

15. Taenzer AH, Clark Ct, Kovarik WD. Experience with 724 epidurograms for epidural catheter placement in pediatric anesthesia. Reg Anesth Pain Med. 2010;35(5):432-435.

16. Tsui BC, Gupta S, Finucane B. Confirmation of epidural catheter placement using nerve stimulation. Can J Anaesth. 1998;45(7):640-644.

17. Tsui BC, Seal R, Koller J, Entwistle L, Haugen R, Kearney R. Thoracic epidural analgesia via the caudal approach in pediatric patients undergoing fundoplication using nerve stimulation guidance. Anesth Analg. 2001;93(5):1152-1155.

18. Chawathe MS, Jones RM, Gildersleve CD, Harrison SK, Morris SJ, Eickmann C. Detection of epidural catheters with ultrasound in children. Paediatr Anaesth. 2003;13(8):681-684.

19. Roberts SA, Galvez I. Ultrasound assessment of caudal catheter position in infants. Paediatr Anaesth. 2005; 15(5):429-432.

20. Rapp HJ, Folger A, Grau T. Ultrasound-guided epidural catheter insertion in children. Anesth Analg. 2005;101(2):333-339.

21. Willschke H, Marhofer P, Bosenberg A, Johnston S, Wanzel O, Sitzwohl C, Kettner S, et al. Epidural catheter placement in children: comparing a novel approach using ultrasound guidance and a standard loss-of-resistance technique. Br J Anaesth. 2006;97(2):200-207.

22. Ponde VC, Bedekar VV, Desai AP, Puranik KA. Does ultrasound guidance add accuracy to continuous caudalepidural catheter placements in neonates and infants? Paediatr Anaesth. 2017;27(10):1010-1014.

23. Bachman SA, Taenzer AH. Thoracic caudal epidural catheter localization using ultrasound guidance. Paediatr Anaesth. 2020;30(2):194-195.

24. Tsui BC, Suresh S. Ultrasound imaging for regional anesthesia in infants, children, and adolescents: a review of current literature and its application in the practice of neuraxial blocks. Anesthesiology. 2010;112(3):719-728.

25. Walker BJ, Long JB, Sathyamoorthy M, Birstler J, Wolf $\mathrm{C}$, Bosenberg AT, Flack SH, et al. Complications in pediatric regional anesthesia: an analysis of more than 100,000 blocks from the pediatric regional anesthesia network. Anesthesiology. 2018;129(4):721-732.

26. Simpao AF, Galvez JA, Wartman EC, England WR, Wu L, Rehman MA, Ngo TV. The migration of caudally threaded thoracic epidural catheters in neonates and in- 
fants. Anesth Analg. 2019;129(2):477-481.

27. Dontukurthy S, Tobias JD. Update on local anesthetic toxicity, prevention and treatment during regional anesthesia in infants and children. J Pediatr Pharmacol Ther. 2021;26(5):445-454.

28. Peutrell JM, Holder K, Gregory M. Plasma bupivacaine concentrations associated with continuous extradural infusions in babies. Br J Anaesth. 1997;78(2):160-162.

29. Henderson K, Sethna NF, Berde CB. Continuous caudal anesthesia for inguinal hernia repair in former preterm infants. J Clin Anesth. 1993;5(2):129-133.
30. Ross EL, Reiter PD, Murphy ME, Bielsky AR. Evaluation of prolonged epidural chloroprocaine for postoperative analgesia in infants. J Clin Anesth. 2015;27(6):463469.

31. Cladis FP, Litman RS. Transient cardiovascular toxicity with unintentional intravascular injection of 3\% 2-chloroprocaine in a 2-month-old infant. Anesthesiology. 2004;100(1):181-183.

32. Hernandez MA, Boretsky K. Chloroprocaine: local anesthetic systemic toxicity in a 9-month infant with paravertebral catheter. Paediatr Anaesth. 2016;26(6):665-666. 